\title{
Altered spontaneous brain activity patterns in diabetic patients with vitreous hemorrhage using amplitude of low-frequency fluctuation: A resting-state fMRI study
}

\author{
WEN-QING SHI ${ }^{1 *}$, LI-YING TANG ${ }^{1,2^{*}}$, QI LIN ${ }^{1}$, BIAO LI $^{1}$, NAN JIANG $^{1,2}$, \\ PEI-WEN ZHU ${ }^{1}$, QING YUAN ${ }^{1}$, LEI YE $^{1}$ and YI SHAO ${ }^{1}$ \\ ${ }^{1}$ Department of Ophthalmology, Jiangxi Province Clinical Ophthalmology Institute, \\ The First Affiliated Hospital of Nanchang University, Nanchang, Jiangxi 330006; \\ ${ }^{2}$ Fujian Provincial Key Laboratory of Ophthalmology and Visual Science, Department of Ophthalmology, \\ Eye Institute of Xiamen University, Xiang' an Hospital of Xiamen University, \\ Xiamen University School of Medicine, Xiamen, Fujian 361000, P.R. China
}

Received May 24, 2019; Accepted January 8, 2020

DOI: $10.3892 / \mathrm{mmr} .2020 .11294$

\begin{abstract}
The aim of the present study was to assess the local character of spontaneous brain activity in type-2 diabetic patients with vitreous hemorrhage $(\mathrm{VH})$ and its relationship with clinical features via the amplitude of low-frequency fluctuations (ALFF) method. A total of 31 subjects (15 females and 16 males) with type-2 diabetic $\mathrm{VH}$ and 31 normal controls (NCs) with similar characteristics (sex, age and educational level) were recruited in the present study. All subjects underwent resting-state functional magnetic resonance imaging scans. The local character of spontaneous brain activity was assessed using the ALFF method. The difference between the type-2 diabetic patients with $\mathrm{VH}$ and NCs was determined using receiver operating characteristic curves. Pearson's correlation analysis was applied to evaluate the relationship between the mean ALFF values of specific brain areas and related clinical manifestations in type-2 diabetic patients with VH. The ALFF values of type-2 diabetic patients with VH were significantly increased in the right and left cerebellum posterior lobes, left cerebellum posterior lobe/left lingual gyrus and bilateral superior frontal gyrus/left postcentral gyrus, compared with those obtained for NCs $(\mathrm{P}<0.05)$. By contrast, these values were significantly decreased in the left and right middle frontal gyri, right medial frontal gyrus/left anterior cingulate, right inferior frontal gyrus, right superior
\end{abstract}

Correspondence to: Dr Yi Shao, Department of Ophthalmology, Jiangxi Province Clinical Ophthalmology Institute, The First Affiliated Hospital of Nanchang University, 17 Yongwaizheng Street, Donghu, Nanchang, Jiangxi 330006, P.R. China

E-mail: freebee99@163.com

${ }^{*}$ Contributed equally

Key words: vitreous hemorrhage, diabetes mellitus, amplitude of low-frequency, spontaneous brain activity frontal gyrus, right middle frontal gyrus, right superior frontal gyrus/middle frontal gyrus and left middle frontal gyrus of the former group compared with the $\mathrm{NCs}(\mathrm{P}<0.05)$. Nevertheless, there was no significant association between the mean ALFF values and clinical characteristics in different brain areas. Unusual spontaneous activity occurred in multiple brain areas, which may suggest the neuropathological mechanisms of visual impairment in type-2 diabetic patients with VH.

\section{Introduction}

Diabetes mellitus is a common chronic metabolic disease with increasing prevalence worldwide (1). Diabetes mellitus may lead to eye disease and visual impairment as a result of abnormal blood vessels supplying oxygen and nutrients to the retina (2) Patients with diabetes occasionally develop fragile blood vessels in the retina that hemorrhage into the vitreous cavity of the orbit; a process termed vitreous hemorrhage (VH), which may lead to sudden severe loss of vision (3). $\mathrm{VH}$ is a sign of advanced diabetic eye disease (1); the incidence of VH is $\sim 7$ cases per 100,000 individuals worldwide and is one of the most common causes for the development of subacute or acute visual disorders (3). VH usually occurs suddenly without any pain and although the symptoms vary, they usually include sudden appearance of spots, sudden blurring of vision or even blindness (4). Mild VH can resolve spontaneously, whereas severe $\mathrm{VH}$ is treated through vitrectomy (5).

Since its development in the 1970s and 1980s, magnetic resonance imaging (MRI) has proven to be a useful non-invasive technique in diagnostic medicine and biomedical research, especially for evaluating the structure, function and neurochemical properties of the brain (6). Functional MRI (fMRI) detects brain activity by measuring changes related to blood flow (7). Researchers can analyze cerebral blood flow and metabolism to explore the activation of specific regions of the brain, such as the visual pathways from the retina to the cortex and the spatial organization of the brain, and these analyses can assist in elucidating the pathogenesis of eye diseases $(8,9)$. 
The amplitude of low-frequency fluctuations (ALFF) is an indicator of regional spontaneous neuronal activity in blood oxygenation level-dependent signals at low frequencies, based on the square root integral of the power spectrum (10). ALFF is widely regarded as a highly accurate and sensitive measurement method that provides an index for evaluating spontaneous neural activity (10). Previous studies have successfully used ALFF to assess the neurological status of retinal detachment (11), acute eye pain (12), primary angle-closure glaucoma (13) and strabismus with amblyopia (9). To the best of our knowledge, the present study is the first to assess the intrinsic brain activity in type-2 diabetic patients with $\mathrm{VH}$ and normal controls (NCs), as well as the correlation between the intrinsic brain activity and clinical manifestations, using the ALFF method. In the present study, it was hypothesized that $\mathrm{VH}$ in patients with type- 2 diabetes may induce abnormal activity in the visual cortex.

\section{Materials and methods}

Subjects. A total of 31 type-2 diabetic patients with VH (sex, 16 males and 15 females; mean age, 56.03 years; age range, $56.03 \pm 4.61$ years) were recruited from June 2017 to September 2018 in the present study. Of these patients, 15 (eight males and seven females) cases were caused by type- 2 diabetic retinopathy and 16 (eight males and eight females) cases were caused retinal vein occlusion. The inclusion criteria for subjects were: i) Diabetes; ii) VH caused by type-2 diabetic retinopathy or retinal vein occlusion (Fig. 1); iii) absence of bilateral ocular diseases (for example, retinal degeneration, optic neuritis, strabismus, amblyopia, cataracts and glaucoma); and (4) normal vision in the affected eyes prior to sudden loss of vision. The exclusion criteria for the subjects were: i) History of ophthalmic surgery (for example, scleral buckle or vitreous and glaucoma surgery); ii) VH with ocular trauma; and iii) history of systemic diseases (for example, heart disease, hypertension and psychiatric diseases.

An equal number of NCs (sex, 16 males and 15 females; total of 31; mean age, 56.48 years; age range, $56.48 \pm 4.29$ years) with similar characteristics (for example, educational level, sex and age) to patients of the VH group were recruited from June 2017 to September 2018 to the present study. The inclusion criteria were: i) Absence of brain parenchymal malformation reported through MRI; ii) best corrected visual acuity (BCVA) $\geq 1.0$, without ocular diseases; iii) absence of serious diseases (for example, heart disease, hypertension and psychiatric diseases), except type-2 diabetes; iv) ability to undergo MRI examination; and v) presence of diabetes without $\mathrm{VH}$.

The Ethics Committee of the First Affiliated Hospital of Nanchang University approved the present study. All methods complied with the Declaration of Helsinki. Each subject provided written informed consent and voluntarily participated after being informed of the purpose, content and potential risks of the present study.

Anxiety and depression score assessment. The scores for anxiety and depression were obtained via questionnaires using the self-rating depression scale (14) and Hamilton anxiety scale (15), respectively. Higher scores on these scales indicated a higher level of anxiety or depression (16).
MRI parameters. MRI scanning was conducted using a 3-Tesla MRI scanner (Trio; Siemens Healthineers). The whole-brain T1-weights were obtained via magnetization-prepared gradient echo images using parameters described in a previous study (13). The duration of the entire scanning sequence was $15 \mathrm{~min}$.

fMRI data analysis. The functional diagrams were analyzed as previously described (9). Briefly, the data were initially filtered through MRIcro software (www.mricro.com) (17) and subsequently the rest of the images were pre-processed using Statistical Parametric Mapping (SPM; http://www.fil.ion.ucl. ac.uk/spm; The MathWorks, Inc.) and the Data Processing Assistant for rs-fMRI software (DPARSFA; version 4.0; http://rfmri.org/DPARSF; Institute of Psychology, Chinese Academy of Sciences). The first ten volumes from each subject were discarded due to the signal reaching equilibrium and the participants' adaptation to the scanning noise. After that, the head motion artifacts were corrected, and the interference effect was eliminated using linear regression. Finally, the data were standardized to meet the space criteria defined by the Montreal Neurological Institute (18).

ALFF analysis. The RESTing-state fMRI data analysis Toolkit (REST) toolkit (19) was applied to divide the brain areas with varying ALFF values of type- 2 diabetic patients with $\mathrm{VH}$ and NCs into regions of interest (ROI). The mean ALFF value of each ROI was obtained by averaging each primitive ALFF value on all voxels. A linear correlation analysis was performed to assess the association between the mean ALFF values of each ROI and the behavioral performance in type-2 diabetic patients with $\mathrm{VH}$.

Statistical analysis. SPSS software (version 22.0; IBM Corp.) was used to analyze the cumulative clinical variables between the $\mathrm{NC}$ and $\mathrm{VH}$ groups by performing $\chi^{2}$ tests for categorical data and independent Student's t-tests for continuous data. $\mathrm{P}<0.05$ was considered to indicate a statistically significant difference. Data are presented as the mean \pm SEM.

The difference in the voxel level between the two groups was studied using the REST toolkit with a two-sample t-test. The statistical threshold for the voxel level was set to $\mathrm{P}<0.05$ using Gaussian random field theory for multiple comparisons. AlphaSim was calibrated with $\mathrm{P}<0.01$ and a cluster size $>0.40$ voxels.

The mean ALFF values in various brain areas of type-2 diabetic patients with $\mathrm{VH}$ and NCs were classified using receiver operating characteristic (ROC) curves, which were performed using SPSS software (version 22.0; IBM Corp.).

Pearson's correlation analysis was performed using GraphPad Prism (version 7.0; GraphPad Software, Inc.) to assess the association between the mean ALFF values of multiple brain regions and related behavioral performances in the VH group.

\section{Results}

Demographics and measurements of vision. The $\mathrm{VH}$ and NC groups did not demonstrate significant differences in terms of age $(\mathrm{P}=0.967)$ and body weight $(\mathrm{P}=0.906)$. However, 
Table I. Demographics and clinical measurements by group.

\begin{tabular}{lcccc}
\hline Condition & VH & NC & t-value & P-value \\
\hline Male/female & $16 / 15$ & $16 / 15$ & N/A & $>0.990$ \\
Age (years) & $56.030 \pm 4.610$ & $56.480 \pm 4.290$ & 0.041 & 0.967 \\
Weight (kg) & $60.480 \pm 3.830$ & $61.100 \pm 3.490$ & 0.118 & 0.906 \\
Handedness & $31 \mathrm{R}$ & $31 \mathrm{R}$ & $\mathrm{N} / \mathrm{A}$ & $>0.990$ \\
Duration of VH (days) & $24.150 \pm 20.970$ & $\mathrm{~N} / \mathrm{A}$ & $\mathrm{N} / \mathrm{A}$ & $\mathrm{N} / \mathrm{A}$ \\
Best-corrected VA-right eye & $0.640 \pm 0.050$ & $1.070 \pm 0.030$ & -7.771 & $<0.001$ \\
Best-corrected VA-left eye & $0.660 \pm 0.060$ & $1.06 \pm 0.020$ & -6.231 & $<0.001$
\end{tabular}

Data are presented as mean \pm SEM. VH, vitreous hemorrhage; NC, normal control; N/A, not applicable; R, right; VA, visual acuity.

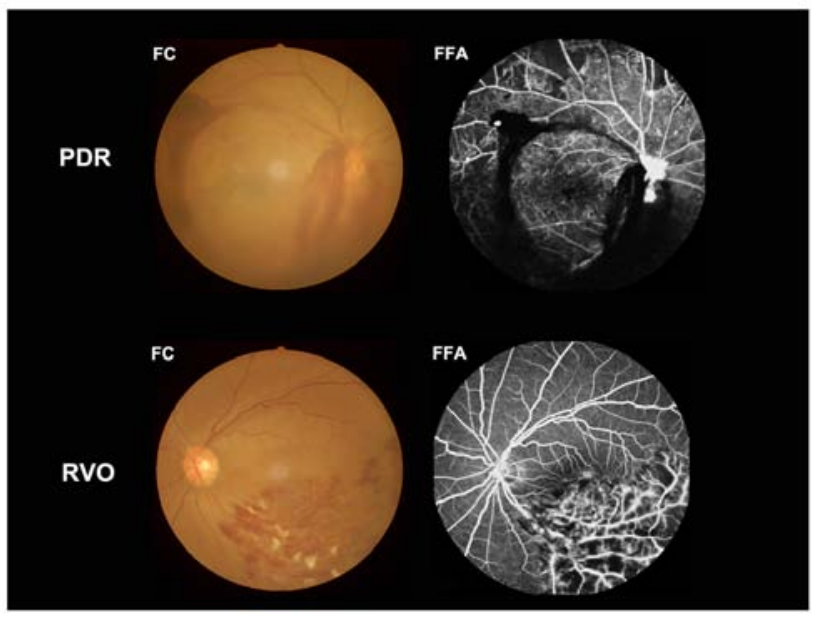

Figure 1. Example of vitreous hemorrhage caused by PDR and RVO observed using a FC and FFA. PDR, proliferative diabetic retinopathy; RVO, retinal vein occlusion; FC, fundus camera; FFA, fluorescence fundus angiography.

a significant difference was observed between the $\mathrm{VH}$ and NC groups for BCVA-right $(\mathrm{P}<0.001)$ and BCVA-left $(\mathrm{P}<0.001)$. In addition, the mean duration of $\mathrm{VH}$ was $24.15 \pm 20.97$ days (Table I).

Differences in ALFF. The ALFF values in the VH group were significantly higher in the bilateral cerebellum posterior lobe (CPL), left CPL/left lingual gyrus (LG) and bilateral superior frontal gyrus (SFG)/left postcentral gyrus (PG) compared with those obtained for the NC group ( $\mathrm{P}<0.05$; Fig. 2 and Table II). Additionally, certain brain areas in the $\mathrm{VH}$ group displayed notably lower ALFF values, including the right middle frontal gyrus (MFG), right inferior frontal gyrus (IFG), right MFG/left anterior cingulate (AC), left MFG-1, right SFG, right MFG, right SFG/MFG and left MFG-2 (Fig. 2 and Table II). Changes in the mean ALFF values between the $\mathrm{VH}$ and $\mathrm{NC}$ groups are presented in Fig. 3. Nevertheless, no correlation was observed between the ALFF values of different brain areas and their manifestations in type-2 diabetic patients with $\mathrm{VH}(\mathrm{P}>0.05)$.

Correlation analysis. In the VH group, the mean ALFF signal values of the right MFG/left AC were negatively correlated with the anxiety score $(\mathrm{r}=-0.906 ; \mathrm{P}<0.0001)$ and depression score ( $\mathrm{r}=-0.854$; $\mathrm{P}<0.0001$; Fig. $4 \mathrm{~A}$ and $\mathrm{B})$. Notably, in the $\mathrm{VH}$ group, BCVA of the contralateral eye was positively correlated with the mean ALFF signal values of the left MFG ( $r=0.634$; $\mathrm{P}<0.0001)$ and right MFG ( $\mathrm{r}=0.494 ; \mathrm{P}=0.004$; Fig. $4 \mathrm{C}$ and $\mathrm{D})$.

ROC curves. It was hypothesized that the difference in ALFF values may serve as a potential diagnostic marker for differentiating type-2 diabetic patients with $\mathrm{VH}$ from NCs. To verify this hypothesis, a ROC curve was constructed by collecting the mean ALFF values of various brain areas in type-2 diabetic patients with VH. An area under the curve (AUC) value of 0.5-0.7 and 0.7-0.9 represented lower and higher accuracy, respectively, of the ALFF value in the different areas of the brain as diagnostic markers. The AUC values of the ALFF values in each area were calculated. The areas of the brain with ALFF values that displayed high accuracy as diagnostic markers (VHs > NCs) were as follows: Right CPL (0.893; $\mathrm{P}<0.001)$, left CPL (0.823; $\mathrm{P}<0.001)$, left CPL/left LG (0.856; $\mathrm{P}<0.001)$ and bilateral SFG/left PG (0.839; $\mathrm{P}<0.001$; Fig. 5A). Contrastingly, the areas of the brain with ALFF values that displayed lower accuracy as diagnostic markers ( VHs $<\mathrm{NCs}$ ) were as follows: Right MFG-1 (0.847; P<0.001), right IFG (0.837; $\mathrm{P}<0.001)$, right $\mathrm{MFG} /$ left $\mathrm{AC}(0.890 ; \mathrm{P}<0.001)$, left MFG-1 (0.868; $\mathrm{P}<0.001)$, right SFG (0.864; $\mathrm{P}<0.001)$, right MFG-2 (0.832; $\mathrm{P}<0.001)$, right SFG/MFG (0.781; $\mathrm{P}<0.001)$ and left MFG-2 (0.850; $\mathrm{P}<0.001$; Fig. 5B).

Relationship between MRI and VH. Based on the results of the present study, the hypothesized mechanism by which intracerebral hemorrhage may cause $\mathrm{VH}$ was that the brain regions associated with the processing of emotions may promote abnormal neural activities. Subsequently, the abnormal neural activities may lead to cerebral hemorrhage, thus promoting $\mathrm{VH}$, impaired visual function and mood swings, initiating a vicious circle (Fig. 6).

Schematic diagram of the mean ALFF values of altered brain regions in the $V H$ group. ALFF values of the following regions in diabetic patients with $\mathrm{VH}$ were increased to various extents compared with those obtained for NCs: (1) Cerebellum posterior lobe. L/lingual gyrus. L (BA 6; $\mathrm{t}=7.0388$ ), (3) cerebellum posterior lobe. L ( $\mathrm{t}=5.7220)$, (5) cerebellum posterior lobe. $\mathrm{R}(\mathrm{t}=5.1342)$, and (6) cerebellum posterior lobe. L/lingual gyrus. L (BA 18; $t=5.0742$ ). By contrast, the ALFF values of the following regions were decreased: (2) Middle frontal 


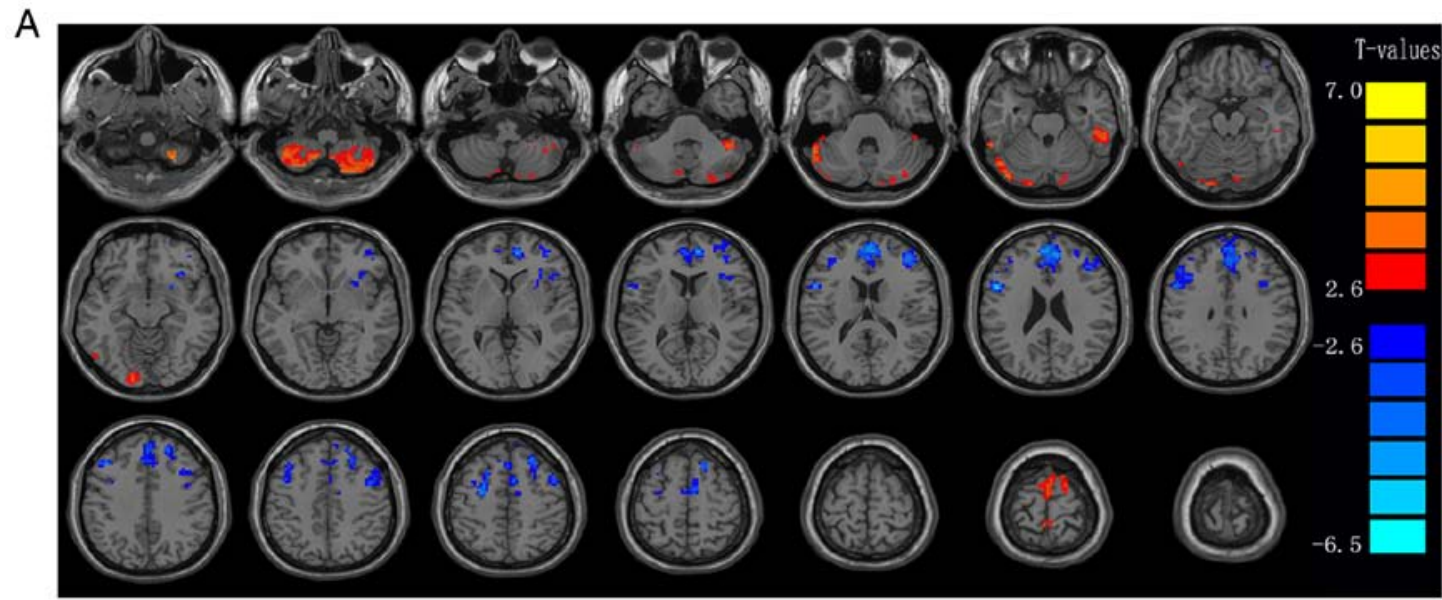

B

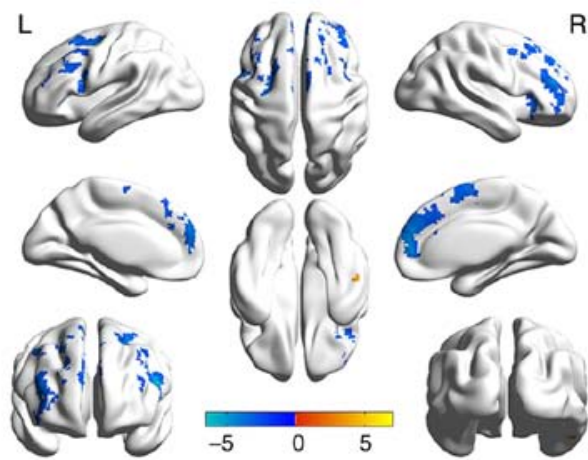

C

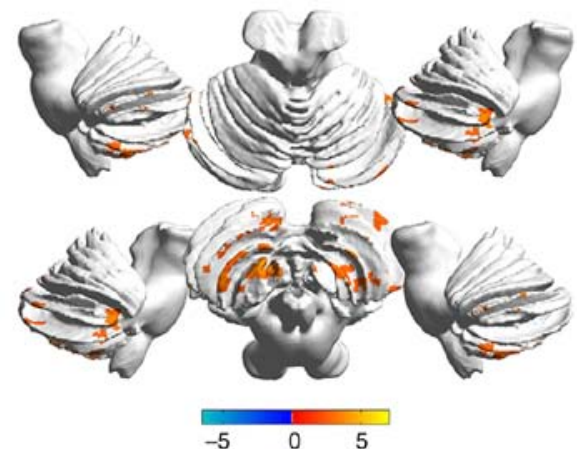

Figure 2. Spontaneous brain activity in diabetic patients with VH vs. NCs. (A) Different ALFF regions between the VH and NC groups. (B) Differences of brain activity in the cerebrum. (C) Differences of brain activity in the cerebellum. Compared with HC, red represents the brain areas with increased ALFF, and blue represents the brain areas with decreased ALFF in patients with VH. $\mathrm{P}<0.01$ for multiple comparisons using Gaussian Random Field theory (AlphaSim corrected; cluster $>40$ voxels; $\mathrm{P}<0.01$ ). ALFF, amplitude of low-frequency fluctuation; VH, vitreous hemorrhage; NCs, normal controls; R, right; L, left.

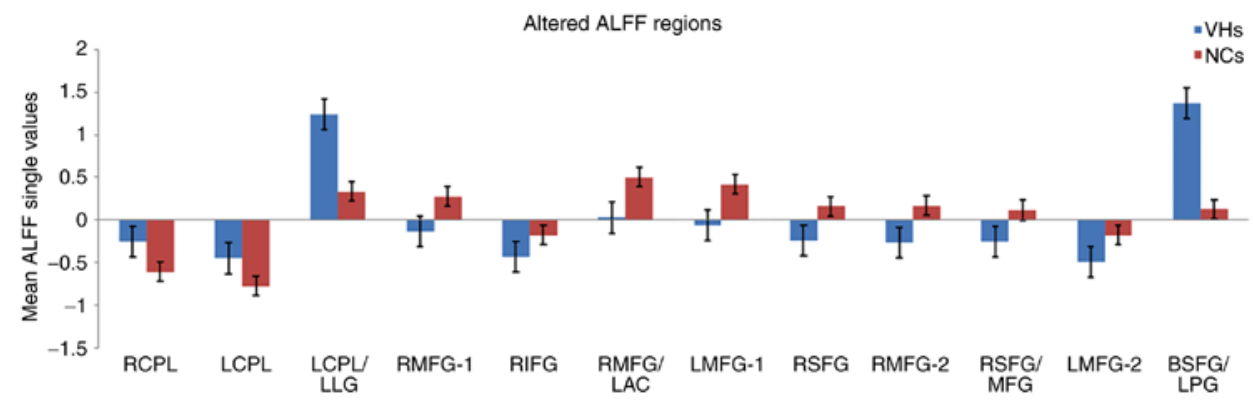

Figure 3. Mean ALFF values between the VH and NC groups in the different regions of the brain. ALFF, amplitude of low-frequency fluctuation; VH, vitreous hemorrhage; NC, normal control; RCPL, right cerebellum posterior lobe; LCPL, left cerebellum posterior lobe; LLG, left lingual gyrus; RMFG, right middle frontal gyrus; RIFG, right inferior frontal gyrus; LAC, left anterior cingulate; LMFG, left middle frontal gyrus; RSFG, right superior frontal gyrus; MFG, middle frontal gyrus; BSFG/LPG, bilateral superior frontal gyrus/left postcentral gyrus.

gyrus. L (BA 10; t=-6.5002), (4) medial frontal gyrus. R (BA 9; $\mathrm{t}=-5.2619)$, (7) middle frontal gyrus. L (BA 10; $\mathrm{t}=-4.6431$ ), (8) superior frontal gyrus. R (BA 6; $t=-4.5000$ ), (9) middle frontal gyrus. R (BA 10; $\mathrm{t}=-4.4753$ ), (10) superior frontal gyrus. R/middle frontal gyrus (BA 10; $\mathrm{t}=-3.8568$ ), (11) middle frontal gyrus. R (BA 10; $t=-3.8469)$ and (12) inferior frontal gyrus. R (BA 45; t=-3.5367) (Fig. 7).

\section{Discussion}

VH is defined as the presence of extravasated blood in the vitreous humor (3). Cerebral hemorrhages, such as aneurysmal subarachnoid hemorrhages, may cause VH (20). In addition, these hemorrhagic brain regions are associated with emotional processing, which may lead to abnormal neurological activity.

fMRI is a technique for measuring brain activity by detecting blood flow-related changes, that has been increasingly used in research to improve understanding of the function of the healthy brain and the disruption of normal cerebral function by disease (21). The ALFF method is used in resting-state fMRI to detect and quantify the activation sites in the brain, as well as to determine the local synchronization of spontaneous fMRI signals (22). Alterations in the mean ALLF values have been found in a variety of ophthalmic 
Table II. Brain areas with significantly different amplitude of low-frequency fluctuations values between VH and NC groups.

A, VHs $>\mathrm{NCs}$

\begin{tabular}{|c|c|c|c|c|c|c|}
\hline \multirow[b]{2}{*}{ Brain regions } & \multicolumn{3}{|c|}{ MNI coordinates } & \multirow[b]{2}{*}{ BA } & \multirow[b]{2}{*}{ Peak voxels } & \multirow[b]{2}{*}{ t-value } \\
\hline & $\mathrm{X}$ & $\mathrm{Y}$ & $\mathrm{Z}$ & & & \\
\hline RCPL & 27 & -66 & -57 & l & 446 & 5.1342 \\
\hline LCPL & -42 & -54 & -57 & l & 185 & 5.7220 \\
\hline RCPL/LCPL & -54 & -51 & -30 & 18 & 244 & 5.0742 \\
\hline BSFG/LPG & -3 & -9 & 78 & 6 & 340 & 7.0388 \\
\hline
\end{tabular}

$\mathrm{B}, \mathrm{VHs}<\mathrm{NCs}$

\begin{tabular}{|c|c|c|c|c|c|c|}
\hline \multirow[b]{2}{*}{ Brain regions } & \multicolumn{3}{|c|}{ MNI coordinates } & \multirow[b]{2}{*}{$\mathrm{BA}$} & \multirow[b]{2}{*}{ Peak voxels } & \multirow[b]{2}{*}{ t-value } \\
\hline & $X$ & $\mathrm{Y}$ & $\mathrm{Z}$ & & & \\
\hline RMFG & 39 & 42 & 18 & 10 & 213 & -4.4753 \\
\hline RIFG & 33 & 27 & 0 & 45 & 92 & -3.5367 \\
\hline RMFG/LAC & 3 & 51 & 15 & $9 / 32$ & 610 & -5.2619 \\
\hline LMGF & -51 & 9 & 21 & 10 & 283 & -6.5002 \\
\hline RSFG & 24 & 30 & 51 & 6 & 156 & -4.5000 \\
\hline RMFG & 48 & 6 & 54 & 10 & 117 & -3.8469 \\
\hline RSFG/MFG & 6 & -3 & 60 & 10 & 97 & -3.8568 \\
\hline LMFG & -27 & -6 & 51 & 10 & 94 & -4.6431 \\
\hline
\end{tabular}

The statistical threshold was set at the voxel level with $\mathrm{P}<0.05$ for multiple comparisons using Gaussian Random Field theory $(\mathrm{z}>2.3, \mathrm{P}<0.01$, cluster $>40$ voxels, AlphaSim corrected). VH, vitreous hemorrhage; NC, normal control; MNI, Montreal Neurological Institute; BA, Brodmann Area; RCPL, right cerebellum posterior lobe; LCPL, left cerebellum posterior lobe; RCPL/LCPL, right cerebellum posterior lobe/left cerebellum posterior lobe; BSFG/LPG, bilateral superior frontal gyrus/left postcentral gyrus; RMFG, right middle frontal gyrus; RIFG/LAC, right inferior frontal gyrus/left anterior cingulate; LMFG, left middle frontal gyrus; RSFG, right superior frontal gyrus; RMFG, right middle frontal gyrus; RSFG/MFG, right superior frontal gyrus/middle frontal gyrus; LMFG, left middle frontal gyrus.

diseases $(13,23)$, suggesting that this method may be helpful in the diagnosis and treatment of such diseases (Table III).

To the best of our knowledge, the present study is the first to assess alterations in the inherent activity patterns in different regions of the brain of type- 2 diabetic patients with VH vs. NCs, via the ALFF method (Fig. 7). The ALFF values of the bilateral CPL, left CPL/left LG and bilateral SFG/left PG were significantly higher in type-2 diabetic patients with VH vs. NCs $(\mathrm{P}<0.05)$. By contrast, the ALFF values of the right MFG-1, right IFG, right MFG/left AC, left MFG-1, right SFG, right $\mathrm{MFG}-2$, right SFG/MFG and left MFG-2 were notably lower in the $\mathrm{VH}$ group compared to the $\mathrm{NC}$ group $(\mathrm{P}<0.05)$.

The CPL, also termed the new cerebellum, is the part below the primary fissure of the cerebellum (24,25). This region receives input from the brain stem and cortex and plays a vital role in fine motor coordination (26), happiness-related activation $(27,28)$ and saccadic eye movements (29). In addition, dysfunction of the CPL is linked to amblyopia with strabismus (9), congenital comitant strabismus (30) and optic neuritis (31). The present study suggested that type-2 diabetic patients with $\mathrm{VH}$ have elevated ALFF values in the bilateral CPL, indicating abnormal brain activity in this region of the brain. Therefore, it can be speculated that type-2 diabetic $\mathrm{VH}$ may result in motor control dysfunction and depression in patients.

The LG, also termed the occipitotemporal gyrus, is related to visual processing, analysis of logical conditions and encoding of visual memory $(32,33)$. In a previous study, patients with primary open-angle glaucoma exhibited decreased ALFF values in the right LG compared with NCs (34). However, in the present study it was revealed that the ALFF values in the left CPL/left LG of type-2 diabetic patients with VH increased, indicating that $\mathrm{VH}$ may disrupt vision, logical analysis and visual memory function in the brain.

The PG is located in the lateral parietal lobe of the brain and plays a key role in sensory function (35). In previous studies, the ALFF values in patients with primary open-angle glaucoma decreased, whereas those of patients with acute eye pain increased $(12,34)$. The results of the present study suggested that ALFF values increased in the bilateral SFG/left PG of type-2 diabetic patients with $\mathrm{VH}$, suggesting that $\mathrm{VH}$ may affect the sensory functions of such patients.

The medial frontal gyrus (MFG) lies in the middle of the frontal gyrus, along with the frontal eye fields, playing a vital role in the regulation of eye movement $(36,37)$. Previous studies have shown that ocular muscle dysfunction may occur 

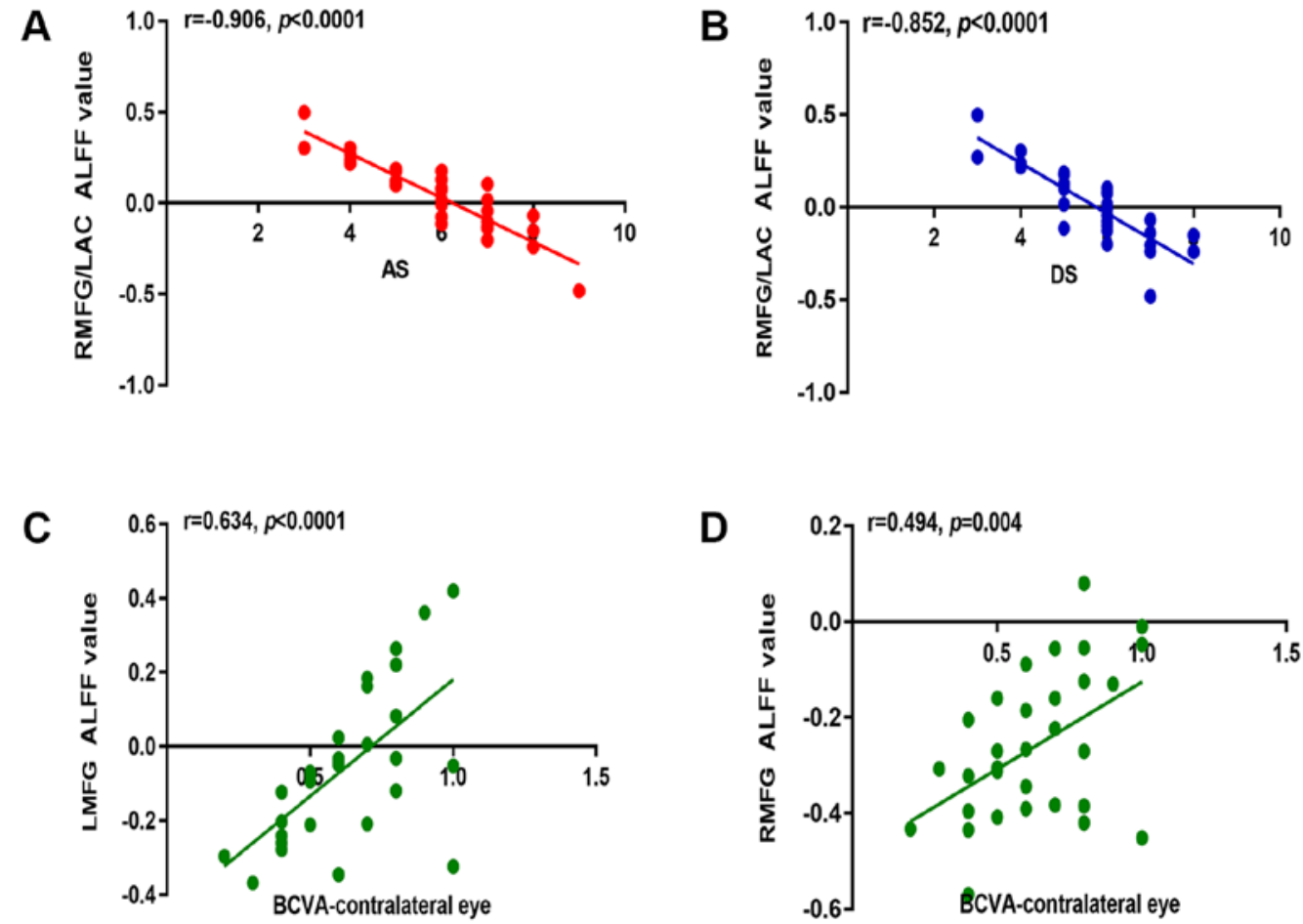

Figure 4. Correlations between the mean ALFF value and behavioral performances in the different areas of the brain. (A) AS in the VH group displayed a negative correlation with the mean ALFF value of the RMFG/LAC ( $r=-0.906 ; \mathrm{P}<0.0001)$. (B) DS in the VH group displayed a negative correlation with the mean ALFF value of the RMFG/LAC ( $\mathrm{r}=-0.852 ; \mathrm{P}<0.0001)$. In the VH group, BCVA of the contralateral eye displayed a positive correlation with the mean ALFF value of $(\mathrm{C})$ the $\mathrm{LMFG}(\mathrm{r}=0.634 ; \mathrm{P}<0.0001)$ and $(\mathrm{D})$ the $\mathrm{RMFG}(\mathrm{r}=0.494 ; \mathrm{P}=0.004)$. $r$, Pearson's correlation coefficient; ALFF, amplitude of low-frequency fluctuation; AS, anxiety score; RMFG, right middle frontal gyrus; LAC, left anterior cingulate; DS, depression score; BCVA, best-corrected visual acuity; LMFG, left middle frontal gyrus.
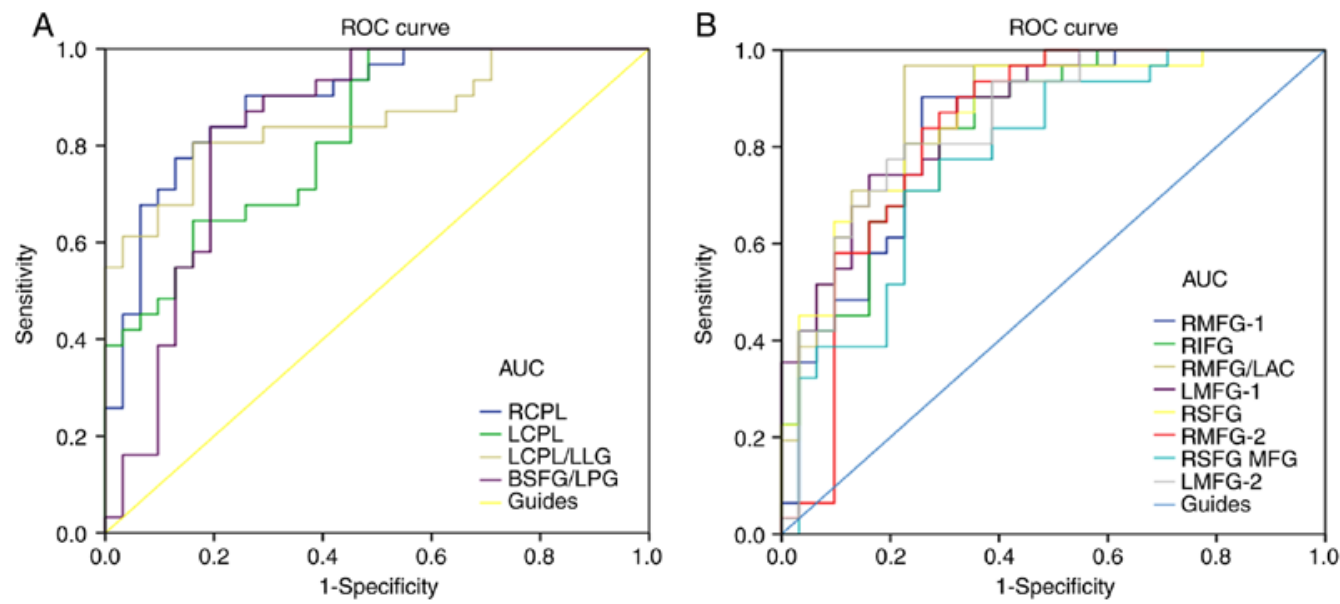

Figure 5. ROC curve analysis of the mean ALFF values of the altered brain regions in VH. (A) The areas under the ROC curve were: RCPL 0.893, (P<0.001; 95\% CI, 0.814-0.972), LCPL 0.823 ( $\mathrm{P}<0.001 ; 95 \%$ CI, 0.723-0.923), LCPL/LLG 0.856 (P<0.001; 95\% CI, 0.761-0.951) and BSFG/LPG 0.839 (P<0.001; 95\% CI, 0.732-0.945). (B) The areas under the ROC curve were: RMFG-1 0.847 (P<0.001; 95\% CI, 0.748-0.946), RIFG 0.837 (P<0.001; 95\% CI, 0.739-0.934), RMFG/LAC 0.890 ( $\mathrm{P}<0.001 ;$ 95\% CI, 0.807-0.972), LMFG-1 0.868 (P<0.001; 95\% CI, 0.782-0.954), RSFG 0.864 (P<0.001; 95\% CI, 0.773-0.954), RMFG-2 0.832 (P<0.001; 95\% CI, 0.724-0.941), RSFG/MFG 0.781 ( $\mathrm{P}<0.001$; 95\% CI, 0.666-0.897) and LMFG-2 0.850 ( $\mathrm{P}<0.001$; 95\% CI, 0.754-0.947). ROC, receiver operating characteristic; ALFF, amplitude of low-frequency fluctuation; RCPL, right cerebellum posterior lobe; CI, confidence interval; LCPL, left cerebellum posterior lobe; LLG, left lingual gyrus; BSFG/LPG, bilateral superior frontal gyrus/left postcentral gyrus; RMFG, right middle frontal gyrus; RIFG, right inferior frontal gyrus; LAC, left anterior cingulate; LMFG, left middle frontal gyrus; RSFG, right superior frontal gyrus; MFG, middle frontal gyrus; AUC, area under the curve.

in ocular diseases; for example, Kang et al (11) reported that patients with retinal detachment had decreased ALFF values in the right MFG. Tan et al (30) reported that patients with congenital concomitant strabismus had significantly lower ALFF values in the bilateral MFG. Huang et al (17) demonstrated that individuals with optic neuritis had decreased ALFF values in the left MFG. Similar to these findings, the data from the present study suggested that the ALFF value of the bilateral MFG was decreased in type-2 diabetic patients with $\mathrm{VH}$, suggesting that $\mathrm{VH}$ may lead to impairment of the local synchronization of brain activity, which may subsequently lead to visually-related motor dysfunction. 
Table III. Amplitude of low-frequency fluctuations method applied in ophthalmological diseases.

\section{Brain areas}

\begin{tabular}{lll}
\cline { 3 - 3 } Author, year & Disease & UDs $>$ NCs
\end{tabular}

Huang et al, 2015 Optic neuritis

Huang et al,2015 Primary angle-closure glaucoma

Li et al, 2014

Tan et al, 2016

Tan et al, 2016

Li et al, 2016

Pan et al, 2018

Min et al, 2018

Kang et al, 2019
Primary open-angle glaucoma

Congenital comitant strabismus

Unilateral acute open globe injury

Late monocular blindness

Acute eye pain

Strabismus with amblyopia

Retinal detachment
LCPL, RCPL, RITG, LPG, RIT/FG, LFG, LCF, LIPL, $\mathrm{LC}$

RPG

RMFG, RSMA
BCPL, LAG
LC, LMCC, BP
RMFG, LMFG, LSG
RPG, LPG, LC
RSFG, RP, LC, BPG
FSO, ITG

RCPL, RCAL, RP, RIFG, RI, LMFG, LSTG, RSG, BAC/MFG, BP, RSG, RIPL RMFG, LMFG, RSFG, RP, LPG, RAG, LSFG RLG, RITG, LPCG BMFG

No brain region

LCAL, RPG, RC, LPG/PL $\mathrm{LP} / \mathrm{PG}, \mathrm{RP} / \mathrm{PG}, \mathrm{LP}$

LCPL, LT, RT, LMFG

$\mathrm{OL}, \mathrm{MFG}$

UD, unusual disease; NCs, normal controls; LCPL, left cerebellum posterior lobe; RCPL, right cerebellum posterior lobe; RITG, right inferior temporal gyrus; LPG, left parahippocampal gyrus; RIT/FG, right inferior temporal/fusiform gyrus; LFG, left fusiform gyrus; LCF, left calcarine fissure; LIPL, left inferior parietal lobule; LC, left cuneus; RCAL, right cerebellum anterior lobe; RP, right putamen; RIFG, right inferior frontal gyrus; RI, right insula; LMFG, left medial frontal gyrus; LSTG, left superior temporal gyrus; RSG, right supramarginal gyrus; BAC/MFG, bilateral anterior cingulate/medial frontal gyrus; BP, bilateral precuneus; RSG, right supramarginal gyrus; RIPL, right inferior parietal lobule; RPG, right precentral gyrus; RMFG, right middle frontal gyrus; RSFG, right superior frontal gyrus; RP, right precuneus; RAG, right angular gyrus; LSFG, left superior frontal gyrus; RSMA, right supplementary motor area; RLG, Right lingual gyrus; LPCG, left postcentral gyrus; BCPL, bilateral cerebellum posterior lobe; LAG, left angular gyrus; BMFG, bilateral medial frontal gyrus; LMCC, left middle cingulum cortex; BP, bilateral precuneus; LSG, let supramarginal gyrus; LCAL, left cerebellum anterior lobe; RPG, right parahippocampal gyrus; RC, right cuneus; LPG/PL, left precentral gyrus/paracentral lobule; LC, left caudate; LP/PG, left precentral/postcentral gyrus; RP/PG, right precentral/postcentral gyrus; LP, left precuneus; BPG, bilateral precentral gyrus; LT, left thalamus; RT, left thalamus; FSO, frontal superior orbital; ITG, inferior temporal gyrus; OL, occipital lobe; MFG, medial frontal gyrus.

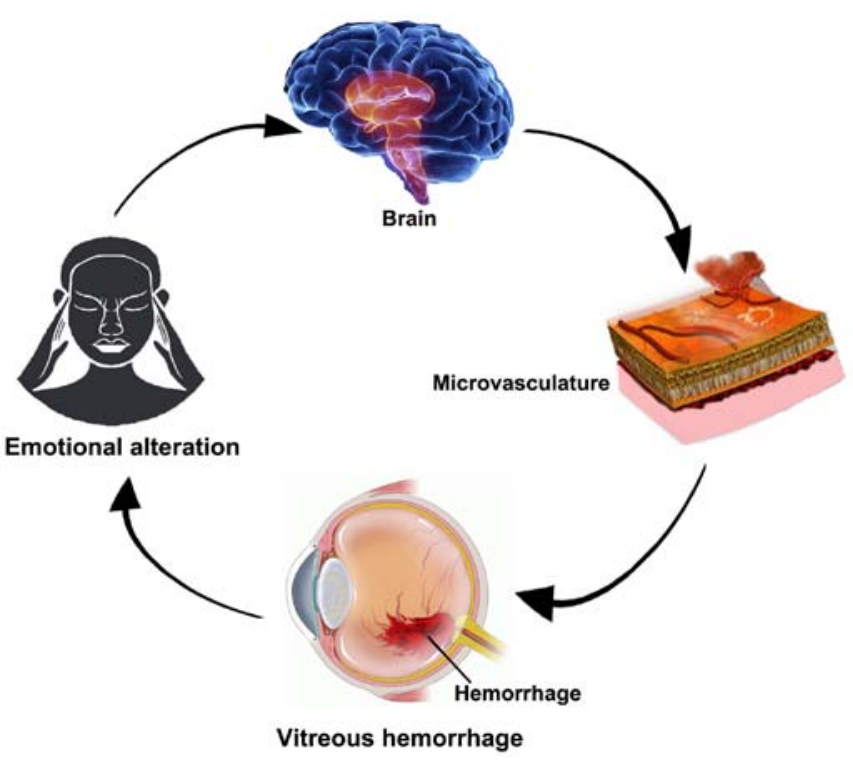

Figure 6. Relationship between magnetic resonance imaging and VH. Intracerebral hemorrhage may cause VH. Once VH occurs, it may affect the function of vision and lead to abnormal nerve activity in areas of the brain that are related to emotional processing. $\mathrm{VH}$, vitreous hemorrhage.

The IFG located in Broca's area of the brain participates in language processing and speech production (38).

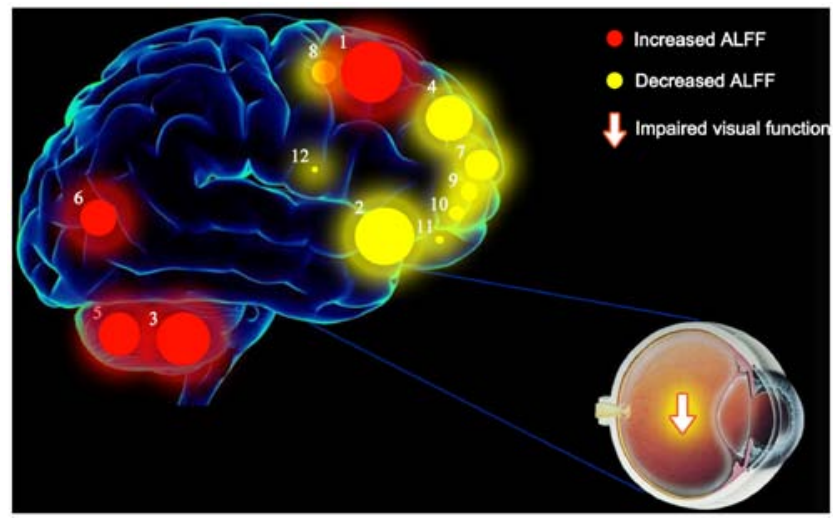

Figure 7. Mean ALFF values of altered brain regions in the VH group. ALFF values of the following regions in diabetic patients with $\mathrm{VH}$ were increased to various extents compared with those obtained for NCs: (1) Cerebellum posterior lobe. L/lingual gyrus. L (BA 6; $\mathrm{t}=7.0388$ ), (3) cerebellum posterior lobe. $\mathrm{L}(\mathrm{t}=5.7220),(5)$ cerebellum posterior lobe. $\mathrm{R}(\mathrm{t}=5.1342)$, and (6) cerebellum posterior lobe. L/lingual gyrus. L (BA 18; $\mathrm{t}=5.0742$ ). By contrast, the ALFF values of the following regions were decreased: (2) Middle frontal gyrus. L (BA 10; $\mathrm{t}=-6.5002)$, (4) medial frontal gyrus. R (BA 9; $\mathrm{t}=-5.2619$ ), (7) middle frontal gyrus. L (BA 10; $\mathrm{t}=-4.6431$ ), (8) superior frontal gyrus. R (BA 6; $\mathrm{t}=-4.5000)$, (9) middle frontal gyrus. R (BA 10; $\mathrm{t}=-4.4753$ ), (10) superior frontal gyrus. $\mathrm{R} /$ middle frontal gyrus (BA $10 ; \mathrm{t}=-3.8568$ ), (11) middle frontal gyrus. R (BA 10; $\mathrm{t}=-3.8469$ ) and (12) inferior frontal gyrus. R (BA 45; $t=-3.5367$ ). The sizes of the spots denote the degree of quantitative changes in the ALFF values between the VH and NC groups. ALFF, amplitude of low-frequency fluctuations; VH, vitreous hemorrhage; NCs, normal controls; R, right; L, left; BA, Brodmann's area. 
Huang et al (17) observed decreased ALFF values in the IFG of patients with optic neuritis. In the present study, a notable decline in ALFF values in the right IFG of type-2 diabetic patients with $\mathrm{VH}$ was observed, suggesting that $\mathrm{VH}$ may cause impairment of language function in this setting.

The SFG is the marginal gyrus, accounting for approximately one-third of the human frontal lobe (39). Using fMRI experiments, Goldberg et al (40) demonstrated that the SFG was related to self-consciousness and coordinated with the movement of the sensory system. It has been previously reported that the ALFF value of the right SFG in strabismus patients with amblyopia is higher than that obtained in NCs (9). By contrast, the present study revealed that the ALFF values in the right SFG were lower in type-2 diabetic patients with VH than in NCs. This finding suggested that VH may have negative effects on self-consciousness and the sensory system.

The AC cortex is located on the anterior part of the cingulate cortex and plays a vital role in various functions, such as the autonomic function of regulation of blood pressure and heart rate, attention and emotional control (41-43). Huang et al (17) revealed that lower ALFF values occurred in the left MFG, left superior temporal gyrus, right inferior parietal lobule and bilateral AC/MFG of patients with optic neuritis. In the present study, type-2 diabetic patients with VH displayed lower ALFF values in the right MFG/left AC, indicating that $\mathrm{VH}$ may cause fluctuations in blood pressure, heart rate, attention and mood.

The main treatment options for severe cases of $\mathrm{VH}$ in patients with diabetes are laser photocoagulation, cryo-coagulation and vitrectomy (44). In the future, fMRI may become a widely used method to observe the treatment of diabetic patients with $\mathrm{VH}$ via the detection of brain activity by measuring changes related to the flow of blood. However, the present study did not compare brain activity in type-2 diabetic patients with $\mathrm{VH}$ prior to and after treatment. The present study reported changes in different brain areas of type-2 diabetic patients with $\mathrm{VH}$ and therefore provides insight for further exploration into the pathogenesis of $\mathrm{VH}$.

The present study revealed changes in spontaneous activity in multiple areas of the brain of type- 2 diabetic patients with $\mathrm{VH}$. These findings may point to altered neural mechanisms present in type-2 diabetic patients with $\mathrm{VH}$ and provide a foundation for conducting further research studies.

\section{Acknowledgements}

Not applicable.

\section{Funding}

The present study was supported by the National Natural Science Foundation of China (grant nos. 81660158, 81460092 and 81400372), the Natural Science Key Project of Jiangxi Province (grant no. 20161ACB21017) and the Health Development Planning Commission Science Foundation of Jiangxi Province (grant no. 20175116).

\section{Availability of data and materials}

The datasets used and/or analyzed during the current study are available from the corresponding author on reasonable request.

\section{Authors' contributions}

WQS and LYT were major contributors; both conceived and designed the experiments, analyzed the data and wrote and revised the manuscript. QL, BL and NJ recruited the healthy controls to the study and performed the MRI experiments. PWZ, QY and LYT collected the data, and YS designed the study and obtained the financial support. All authors read and approved the final manuscript.

\section{Ethics approval and consent to participate}

Thepresentstudy wasperformed underthe approvaloftheMedical Research Ethics Committee of the First Affiliated Hospital of Nanchang University. The methods in the present study were conducted under relevant guidelines and regulations. All participants involved were offered the whole study design and signed the informed consent form.

\section{Patient consent for publication}

Not applicable.

\section{Competing interests}

The authors declare that they have no competing interests.

\section{References}

1. Shaw JE, Sicree RA and Zimmet PZ: Global estimates of the prevalence of diabetes for 2010 and 2030. Diabetes Res Clin Pract 87: 4-14, 2010.

2. Ong SR, Crowston JG, Loprinzi PD and Ramulu PY: Physical activity, visual impairment, and eye disease. Eye (Lond) 32: 1296-1303, 2018.

3. Spraul CW and Grossniklaus HE: Vitreous hemorrhage. Surv Ophthalmol 42: 3-39, 1997.

4. Yoonessi R, Hussain A and Jang TB: Bedside ocular ultrasound for the detection of retinal detachment in the emergency department. Acad Emerg Med 17: 913-917, 2010.

5. Cuevas P, Outeirino LA, Azanza C, Angulo J and GimenezGallego G: Dramatic resolution of vitreous hemorrhage after an intravitreal injection of dobesilate. Mil Med Res 2: 23, 2015.

6. Brown HD, Woodall RL, Kitching RE, Baseler HA and Morland AB: Using magnetic resonance imaging to assess visual deficits: A review. Ophthalmic Physiol Opt 36: 240-265, 2016.

7. Katwal SB, Gore JC, Gatenby JC and Rogers BP: Measuring relative timings of brain activities using fMRI. NeuroImage 66: 436-448, 2013.

8. Conner IP, Odom JV, Schwartz TL and Mendola JD: Monocular activation of V1 and V2 in amblyopic adults measured with functional magnetic resonance imaging. J AAPOS 11: 341-350, 2007.

9. Min YL, Su T, Shu YQ, Liu WF, Chen LL, Shi WQ, Jiang N, Zhu PW, Yuan Q, Xu XW, et al: Altered spontaneous brain activity patterns in strabismus with amblyopia patients using amplitude of low-frequency fluctuation: A resting-state fMRI study. Neuropsychiatr Dis Treat 14: 2351-2359, 2018.

10. Zuo XN, Di Martino A, Kelly C, Shehzad ZE, Gee DG, Klein DF, Castellanos FX, Biswal BB and Milham MP: The oscillating brain: Complex and reliable. Neuroimage 49: 1432-1445, 2010.

11. Kang HH, Shu YQ, Yang L, Zhu PW, Li D, Li QH, Min YL, Ye L, Zhou Q and Shao Y: Measuring abnormal intrinsic brain activities in patients with retinal detachment using amplitude of low-frequency fluctuation: A resting-state fMRI study. Int J Neurosci 129: 681-686, 2019.

12. Pan ZM, Li HJ, Bao J, Jiang N, Yuan Q, Freeberg S, Zhu PW, Ye L, Ma MY, Huang X and Shao Y: Altered intrinsic brain activities in patients with acute eye pain using amplitude of low-frequency fluctuation: A resting-state fMRI study. Neuropsychiatr Dis Treat 14: 251-257, 2018. 
13. Huang X, Zhong YL, Zeng XJ, Zhou F, Liu XH, Hu PH, Pei CG, Shao Y and Dai XJ: Disturbed spontaneous brain activity pattern in patients with primary angle-closure glaucoma using amplitude of low-frequency fluctuation: A fMRI study. Neuropsychiatr Dis Treat 11: 1877-1883, 2015.

14. Zung WW: A self-rating depression scale. Arch Gen Psychiatry 12: 63-70, 1965.

15. Hamilton $\mathrm{M}$ : The assessment of anxiety states by rating. $\mathrm{Br} \mathrm{J}$ Med Psychol 32: 50-55, 1959

16. Zigmond AS and Snaith RP: The hospital anxiety and depression scale. Acta Psychiatr Scand 67: 361-370, 1983.

17. Huang X, Cai FQ, Hu PH, Zhong YL, Zhang Y, Wei R, Pei CG, Zhou FQ and Shao Y: Disturbed spontaneous brain-activity pattern in patients with optic neuritis using amplitude of low-frequency fluctuation: A functional magnetic resonance imaging study. Neuropsychiatr Dis Treat 11: 3075-3083, 2015.

18. Evans AC, Janke AL, Collins DL and Baillet S: Brain templates and atlases. Neuroimage 62: 911-922, 2012.

19. Chang $\mathrm{J}$ and $\mathrm{Yu} \mathrm{R}$ : Acute social stress modulates coherence regional homogeneity. Brain Imaging Behav 13: 762-770, 2019.

20. Farnarier G, Sobrepere G and Croisy A: Syndrome of hematoma of the vitreous body with Terson's spontaneous intracranial hemorrhage. Bull Soc Ophtalmol Fr 8: 556-560, 1955 (In French).

21. Bandettini PA: Twenty years of functional MRI: The science and the stories. NeuroImage 62: 575-588, 2012

22. Zang YF, He Y, Zhu CZ, Cao QJ, Sui MQ, Liang M, Tian LX, Jiang TZ and Wang YF: Altered baseline brain activity in children with ADHD revealed by resting-state functional MRI. Brain Dev 29: 83-91, 2007.

23. Li Q, Huang X, Ye L, Wei R, Zhang Y, Zhong YL, Jiang N and Shao Y: Altered spontaneous brain activity pattern in patients with late monocular blindness in middle-age using amplitude of low-frequency fluctuation: A resting-state functional MRI study. Clin Interv Aging 11: 1773-1780, 2016.

24. Stoodley CJ and Schmahmann JD: Functional topography of the human cerebellum. Handb Clin Neurol 154: 59-70, 2018.

25. Paulin MG: The role of the cerebellum in motor control and perception. Brain Behav Evol 41: 39-50, 1993.

26. Casula EP, Pellicciari MC, Ponzo V, Stampanoni Bassi M, Veniero D, Caltagirone C and Koch G: Cerebellar theta burst stimulation modulates the neural activity of interconnected parietal and motor areas. Sci Rep 6: 36191, 2016.

27. Stoodley CJ and Schmahmann JD: Functional topography in the human cerebellum: A meta-analysis of neuroimaging studies. Neuroimage 44: 489-501, 2009.

28. Schienle A and Scharmuller W: Cerebellar activity and connectivity during the experience of disgust and happiness. Neuroscience 246: 375-381, 2013.

29. Hayakawa Y, Nakajima T, Takagi M, Fukuhara N and Abe $\mathrm{H}$ Human cerebellar activation in relation to saccadic eye movements: A functional magnetic resonance imaging study. Ophthalmologica 216: 399-405, 2002.

30. Tan G, Huang X, Zhang Y, Wu AH, Zhong YL, Wu K, Zhou FQ and Shao Y: A functional MRI study of altered spontaneous brain activity pattern in patients with congenital comitant strabismus using amplitude of low-frequency fluctuation. Neuropsychiatr Dis Treat 12: 1243-1250, 2016.

31. Ru Y, Huang Y, Liu H, Du J, Meng Z, Dou Z, Liu X, Wei RH, Zhang $\mathrm{Y}$ and Zhao S: $\alpha$-Melanocyte-stimulating hormone ameliorates ocular surface dysfunctions and lesions in a scopolamine-induced dry eye model via PKA-CREB and MEK-Erk pathways. Sci Rep 5: 18619, 2015.
32. Mechelli A, Humphreys GW, Mayall K, Olson A and Price CJ: Differential effects of word length and visual contrast in the fusiform and lingual gyri during reading. Proc Biol Sci 267: 1909-1913, 2000

33. Bogousslavsky J, Miklossy J, Deruaz JP, Assal G and Regli F: Lingual and fusiform gyri in visual processing: A clinico-pathologic study of superior altitudinal hemianopia. J Neurol Neurosurg Psychiatry 50: 607-614, 1987.

34. Li T, Liu Z, Li J, Liu Z, Tang Z, Xie X, Yang D, Wang N, Tian J and Xian J: Altered amplitude of low-frequency fluctuation in primary open-angle glaucoma: A resting-state FMRI study. Invest Ophthalmol Vis Sci 56: 322-329, 2014.

35. Kaukoranta E, Hamalainen M, Sarvas J and Hari R: Mixed and sensory nerve stimulations activate different cytoarchitectonic areas in the human primary somatosensory cortex SI. Neuromagnetic recordings and statistical considerations. Exp Brain Res 63: 60-66, 1986.

36. Seitz RJ, Nickel J and Azari NP: Functional modularity of the medial prefrontal cortex: Involvement in human empathy. Neuropsychology 20: 743-751, 2006.

37. Cameron IG, Riddle JM and D'Esposito M: Dissociable roles of dorsolateral prefrontal cortex and frontal eye fields during saccadic eye movements. Front Hum Neurosci 9: 613, 2015.

38. Greenlee JD, Oya H, Kawasaki H, Volkov IO, Severson MA III, Howard MA III and Brugge JF: Functional connections within the human inferior frontal gyrus. J Comp Neurol 503: 550-559, 2007.

39. Shi WQ, Wu W, Ye L, Jiang N, Liu WF, Shu YQ, Su T, Lin Q, Min YL, Li B, et al: Altered spontaneous brain activity patterns in patients with corneal ulcer using amplitude of low-frequency fluctuation: An fMRI study. Exp Ther Med 18: 125-132, 2019.

40. Goldberg II, Harel M and Malach R: When the brain loses its self: Prefrontal inactivation during sensorimotor processing. Neuron 50: 329-339, 2006.

41. Pardo JV, Pardo PJ, Janer KW and Raichle ME: The anterior cingulate cortex mediates processing selection in the stroop attentional conflict paradigm. Proc Natl Acad Sci USA 87: 256-259, 1990.

42. Decety J and Jackson PL: The functional architecture of human empathy. Behav Cogn Neurosci Rev 3: 71-100, 2004.

43. Jackson PL, Brunet E, Meltzoff AN and Decety J: Empathy examined through the neural mechanisms involved in imagining how I feel versus how you feel pain. Neuropsychologia 44: 752-761, 2006

44. El Annan J and Carvounis PE: Current management of vitreous hemorrhage due to proliferative diabetic retinopathy. Int Ophthalmol Clin 54: 141-153, 2014.

45. Tan G, Huang X, Ye L, et al: Altered spontaneous brain activity patterns in patients with unilateral acute open globe injury using amplitude of low-frequency fluctuation: a functional magnetic resonance imaging study. Neuropsychiatric Disease and Treatment 12: 2015-2020, 2016.

This work is licensed under a Creative Commons Attribution-NonCommercial-NoDerivatives 4.0 International (CC BY-NC-ND 4.0) License. 